\title{
Development of TIMPI magnetic nanoformulation for regulation of synaptic plasticity in HIV-I infection
}

\author{
Venkata Subba Rao Atluri* \\ Rahul Dev Jayant* \\ Sudheesh Pilakka- \\ Kanthikeel \\ Gabriella Garcia \\ Thangavel Samikkannu \\ Adriana Yndart \\ Ajeet Kaushik \\ Madhavan Nair \\ Center for Personalized \\ Nanomedicine, Department of \\ Immunology, Herbert Wertheim \\ College of Medicine, Florida \\ International University, Miami, \\ FL, USA \\ *These authors contributed equally \\ to this work
}

This article was published in the following Dove Press journal:

International Journal of Nanomedicine

31 August 2016

Number of times this article has been viewed

\begin{abstract}
Although the introduction of antiretroviral therapy has reduced the prevalence of severe forms of neurocognitive disorders, human immunodeficiency virus (HIV)-1-associated neurocognitive disorders were observed in $50 \%$ of HIV-infected patients globally. The bloodbrain barrier is known to be impermeable to most of antiretroviral drugs. Successful delivery of antiretroviral drugs into the brain may induce an inflammatory response, which may further induce neurotoxicity. Therefore, alternate options to antiretroviral drugs for decreasing the HIV infection and neurotoxicity may help in reducing neurocognitive impairments observed in HIVinfected patients. In this study, we explored the role of magnetic nanoparticle (MNP)-bound tissue inhibitor of metalloproteinase-1 (TIMP1) protein in reducing HIV infection levels, oxidative stress, and recovering spine density in HIV-infected SK-N-MC neuroblastoma cells. We did not observe any neuronal cytotoxicity with either the free TIMP1 or MNP-bound TIMP1 used in our study. We observed significantly reduced HIV infection in both solution phase and in MNP-bound TIMP1-exposed neuronal cells. Furthermore, we also observed significantly reduced reactive oxygen species production in both the test groups compared to the neuronal cells infected with HIV alone. To observe the effect of both soluble-phase TIMP1 and MNP-bound TIMP1 on spine density in HIV-infected neuronal cells, confocal microscopy was used. We observed significant recovery of spine density in both the test groups when compared to the cells infected with HIV alone, indicting the neuroprotective effect of TIMP1. Therefore, our results suggest that the MNP-bound TIMP1 delivery method across the blood-brain barrier can be used for reducing HIV infectivity in brain tissue and neuronal toxicity in HIV-infected patients.
\end{abstract}

Keywords: HIV, neurocognitive disorders, TIMP1, magnetic nanoparticles, blood-brain barrier, neuroprotection

\section{Introduction}

Human immunodeficiency virus (HIV) is a neurotropic virus that enters the brain shortly after infection. It has been reported that HIV enters the central nervous system (CNS) via infected monocytes that traverse the blood-brain barrier (BBB) to replenish perivascular macrophages. Brain macrophages and microglial cells are reported to be the key cells productively infected with HIV, and a few studies have also reported HIV infection in astrocytes, ${ }^{1,2}$ which results in HIV-associated neurocognitive disorders (HAND). ${ }^{3}$ Although very few reports suggest neurons being infected by HIV, ${ }^{4-6}$ but postmortem studies of brains of patients with HAND show decreased synaptic plasticity/ function at various levels. Synaptic plasticity is defined as a change in efficacy/strength of synapses (spine formation and maintenance of synaptic architecture) in response to the external stimuli. Neuronal plasticity dysregulation can be seen at the cellular level
Correspondence: Madhavan Nair

Center for Personalized Nanomedicine, Department of Immunology, Herbert Wertheim College of Medicine, Florida International University, AHC-I, 4I8A, II 200 SW 8th Street, Miami, FL 33199, USA

$\mathrm{Tel}+\mathrm{I} 305348 \mid 493$

Fax + I 305348602 I

Email nairm@fiu.edu 
by decreased spine density and synaptodendritic damage ${ }^{6}$ and at the systemic levels with gray and white matter atrophy $y^{7,8}$ in both cortical and subcortical regions. ${ }^{9,10}$

Matrix metalloproteinases (MMPs) are a family of endopeptidases that play a major role in the degradation of most extracellular matrix proteins, wound healing, and tissue morphogenesis. ${ }^{1-14}$ Tissue inhibitors of metalloproteinases (TIMPs) are a family of proteinase inhibitors that control the activity of MMPs in vivo by binding in 1:1 stoichiometry. ${ }^{15,16}$ Disruption of this balance is seen in various pathological conditions such as neuroAIDS,,${ }^{17}$ Alzheimer's disease, ${ }^{18}$ multiple sclerosis, ${ }^{19}$ brain tumors,${ }^{20}$ epilepsy,${ }^{21}$ and excitotoxic/ neuroinflammatory processes. ${ }^{22}$ In the case of HIV infection, once the HIV enters into the CNS, it leads to neuronal damage, which in turn causes cognitive issues (eg, attention, memory, language, problem solving, and decision-making), and eventually leads to the HAND. Due to such diverse roles in disease advancement, MMPs can serve as a promising therapeutic target, leading to the development of many small-molecule or broad-spectrum MMP inhibitors. But issues like off-target effects, ineffective therapeutic plasma levels, and toxicity (eg, musculoskeletal toxicity) lead to the failure of these compounds at early stages of the clinical trials (eg, studies related to cancer and arthritis). ${ }^{23-26}$ Among all inhibitors, TIMP1 has strong affinity for MMP-9 and holds great therapeutic value in a wide variety of inflammatory and vascular diseases and in cancer. ${ }^{27}$ In our previous studies, we observed that TIMP1 is one of the highly downregulated synaptic plasticity genes in HIV-infected neuronal cells. ${ }^{6}$ TIMP1 also acts as a neuroprotective agent in HIV infection by protecting neurons from apoptosis and by preserving the neuronal architecture. ${ }^{28}$ However, like most proteins, TIMP1 does not cross the BBB and the native form has a short halflife ( $<4$ hours), rapid clearance, and low bioavailability, thus limiting its application as a neuroprotecting agent for CNS diseases. ${ }^{29}$

Nanotechnology is a platform to develop promising and novel drug delivery systems in medicine, and significant research exploring this novel technology has been focused on the delivery of different drugs/proteins/therapeutic agents to brain. Various kinds of drug delivery approaches to deliver drugs across the BBB are being pursued including the use of macrophage-mediated transport, receptor-mediated transport, and use of lipid carrier and tagging drugs to ligands through a carrier. ${ }^{30}$ Unfortunately, in vivo strategies like viral vector upregulation, direct injection into the brain, transnasal, infusion pump-mediated, and transient disruption of BBB lack practical relevance for patient treatment. ${ }^{31-33}$ In clinical trials, systemic side effects are the limiting factors observed while delivering the optimum doses that have therapeutic effects within the brain. ${ }^{34}$ It is necessary to develop innovative technologies to deliver various neurotropic factors across the BBB to reduce the neurotoxic effects observed in various diseases. More recently, due to the intrinsic magnetic properties, magnetic nanoparticles (MNPs) have gained increasing importance owing to the advantage of tracking them through imaging methods. Along with our reports, several other in vitro and in vivo studies have reported the use of magnetically guided drug delivery systems. ${ }^{35-40}$ Previously, our group has shown the application of magnetic nanocarriers for delivering anti-HIV drugs and neuroprotective agents across the BBB using noninvasive external magnetic force and demonstrated that this target-specific CNS strategy can be used for the treatment of neuroAIDS. ${ }^{41,42}$ Recently, we also developed a novel layer-by-layer magnetic nanoformulation (NF) to deliver an anti-HIV drug and a latency reactivating agent across the BBB in a sustained-release manner for 1 week for the treatment of neuroAIDS. ${ }^{43}$ The magnetic platform will help for speedy navigation of the drug-loaded nanocarrier to the target (brain), prevent reticuloendothelial system uptake of the drug, and reduce off-target effects. In the present study, we evaluated the role of TIMP1 protein in neuroprotection by regulating the HIV infection, oxidative stress, and spine density and its implication in prevention of HAND using nanotechnology platform. We synthesized MNP-loaded TIMP1 NF, tested its release kinetics and transmigration across BBB using an in vitro model, and observed the neuroprotective effect of TIMP1 in HIV-1infected neuronal cells.

\section{Materials and methods Synthesis of super paramagnetic iron oxide nanoparticles}

The iron oxide $\left(\mathrm{Fe}_{3} \mathrm{O}_{4}\right)$ magnetic nanoparticles (MNPs) were synthesized according to the coprecipitation method as described previously. ${ }^{43,40}$

\section{Characterization of MNPs}

Synthesized MNPs were characterized for size and shape using transmission electron microscopy (TEM; Phillips CM-200, $200 \mathrm{kV}$ operated at $80 \mathrm{kV}$ ) as described previously. ${ }^{43}$ The hydrodynamic radius, size distribution, and surface charge measurement of TIMP1-loaded and -unloaded MNPs were carried out at $25^{\circ} \mathrm{C}$ using Malvern Zetasizer (dynamic laser scattering, Nano ZS; Malvern Instruments, Inc., Westborough, MA, USA). 


\section{Time kinetics, drug-binding isotherm, and in vitro release studies of TIMPI}

TIMP1 protein was purchased from the Life Technologies (Catalog no 10934-H08H). To determine the time kinetics of direct binding of TIMP1 protein on MNPs, different amounts of MNPs (range: 5-20 $\mu \mathrm{g}$ ) were added to $5 \mu \mathrm{g}$ of TIMP1 and incubated in phosphate-buffered saline (PBS) ( $\mathrm{pH} 7.4)$ for $0,10,20,30$, and 60 minutes at room temperature (RT). The amount of TIMP1 bound (\% binding) to the MNPs was determined by estimating the concentration of TIMP1 in the unbound fraction (supernatant) of the mixture using a UV nanospectrophotometer (Synergy ${ }^{\mathrm{TM}}$ HT Multi-Mode Microplate Reader; BioTek Instrument, Inc., Winooski, VT, USA) at $280 \mathrm{~nm}$. TIMP1 + MNP NF (5 $\mu \mathrm{g}$ MNPs with $5 \mu \mathrm{g}$ TIMP1) release studies were performed in vitro in an Eppendorf tube containing $1,000 \mu \mathrm{L}$ of PBS ( $\mathrm{pH} 7.4$ ) incubated at $37^{\circ} \mathrm{C}$ in an incubator; aliquots of release samples were collected at preset interval times (every 30 minutes) and aliquots of the same volume were replaced with fresh PBS solution to maintain sink condition. The cumulative TIMP1 release percentage was obtained using the amount of TIMP1 released from the total amount of TIMP1 loaded and was determined spectrophotometrically at $\lambda_{\max }$ of $280 \mathrm{~nm}$.

\section{Cell culture and reagents}

SK-N-MC neuroblastoma cells were obtained from American Type Culture Collection (ATCC, Manassas, VA, USA; catalog no HTB-10). HIV-1Ba-L (clade B, macrophage-tropic [R5] virus) was obtained through the AIDS Research and Reference Reagent Program, Division of AIDS, National Institute of Allergy and Infectious Diseases (NIAID), National Institutes of Health (NIH) (Washington DC, USA; AIDS Reagent Program, catalog no 510).

\section{HIV-I infection of human SK-N-MC cells}

An established laboratory protocol was used for infecting the SK-N-MC cells with HIV, ${ }^{6}$ with slight modifications. Briefly, the SK-N-MC $\left(1 \times 10^{5}\right.$ cells $)$ cells were grown overnight in six-well plates in the Dulbecco's Modified Eagle's Medium, and these cells were activated by treating with polybrene $(10 \mu \mathrm{g} / \mathrm{mL})$ for $6-7$ hours before the infection. The cells were infected with optimized concentration (20 ng) of HIV-1 clade B virus overnight, were washed to remove the unbound virus, and were incubated for a further 5 days. On the fifth day, TIMP1 solution or MNP NF containing TIMP1 was added to these cells and incubated for 48 hours. The culture supernatant collected after 7 days of infection was used for the p24 antigen estimation using an enzyme-linked immunosorbent assay (ELISA) kit (catalog no 0801200; ZeptoMetrix Corp., Buffalo, NY, USA). Control cells (without HIV, TIMP1/MNP-TIMP1) were included in the setup of all experiments.

\section{In vitro BBB preparation, validation, and transmigration assay}

The BBB model was prepared as described by Persidsky et $\mathrm{al}^{44}$ and modified by us. ${ }^{43}$ Briefly, in a bicompartmental transwell culture plate (product no 3415; Corning Life Sciences, Reynosa, Mexico), the in vitro BBB model was prepared. An insert with $10 \mu \mathrm{m}$ thick polycarbonate membrane possessing $3.0 \mu \mathrm{m}$ pores separates the upper chamber of this plate from the lower chamber. In a sterile 24-well cell culture plate with the insert, human brain microvascular endothelial cells in the upper chamber and human astrocytes on the underside of lower chamber were grown to confluency. To assess the effect of MNP + TIMP1 NF on integrity of the in vitro BBB model, after transmigration assay, paracellular transport of fluorescein isothiocyanate-dextran (FITC-dextran) was measured. ${ }^{43}$ Briefly, $100 \mathrm{mg} / \mathrm{mL}$ FITCdextran (Sigma-Aldrich Co., St Louis, MO, USA) was added to the upper chamber of the inserts and further incubated for 6 hours. Samples were collected from the bottom chamber after 6 hours and relative fluorescence was measured at an excitation wavelength of $485 \mathrm{~nm}$ and an emission wavelength of $520 \mathrm{~nm}$ using Synergy HT Multi-Mode Microplate Reader instrument (Biotek Instrument, Inc.). FITC-dextran transport was expressed as percentage of FITC-dextran transported across the $\mathrm{BBB}$ into the lower compartment compared to negative control. Intactness of in vitro BBB was determined by measuring the transendothelial electrical resistance (TEER) using Millicell ERS microelectrodes (EMD Millipore, Billerica, MA, USA). On the fifth day of $\mathrm{BBB}$ preparation, when the ideal integrity of the $\mathrm{BBB}$ was achieved (by measuring TEER values), transmigration assay of MNP-bound TIMP1 was performed. MNP + TIMP1 NF was added to the upper chamber of the $\mathrm{BBB}$ preparation and incubated at $37^{\circ} \mathrm{C}$ with/without magnetic force of $(0.08 \mathrm{~T})$ placed externally below the transwell basolateral chamber. MNP + TIMP1 NF was collected from the lower chamber at different time points (ie, 1, 2, 3, and 6 hours) and \% transmigration was analyzed for all the different time points using ammonium thiocyanate-based photometric assay. ${ }^{43}$

\section{Intracellular uptake analysis}

Human neuroblastoma SK-N-MC cells were seeded in sixwell plates at a concentration of $1 \times 10^{6}$ cells. After 24 hours, 
cells were treated with equal quantity of FITC-tagged MNP + TIMP1 NF for 3-24 hours. Cells were rinsed with PBS, and to quantify the cellular uptake of TIMP1-loaded nanoparticle levels, the flow cytometer was used. ${ }^{45}$ These cells were harvested at different time points (3-24 hours) after treatment, and 1 million cells were aliquoted in $12 \times 75 \mathrm{~mm}$ polystyrene falcon tubes (BD Biosciences, San Jose, CA, USA; catalog no 352058) and fixed with Cytofix solution (BD Biosciences). Cells for uptake were analyzed in a flow cytometer (BD Accuri Cytometers Inc., Ann Arbor, MI, USA), and a total of 10,000 events were collected for each sample. The results were analyzed using the FlowJo software (Tree Star Inc., Ashland, OR, USA). Cells were gated based on cells with and without NF controls. Cells positive for NF are shown as colored histograms and compared with controls.

\section{Cytotoxicity assay}

Cytotoxicity of MNPs alone and TIMP1-loaded MNP NF was assessed by MTT cell viability assay using the established protocol. ${ }^{46}$ SK-N-MC cells were seeded in sixwell tissue plates at a density of $1 \times 10^{5}$ cells per well and infected with HIV as explained in the "HIV-1 infection of human SK-N-MC cells" section. After 5 days of infection, $100 \mu \mathrm{L}$ fresh media containing different amounts of $\mathrm{Fe}_{3} \mathrm{O}_{4}$ (5-20 $\mu \mathrm{g}$ ), TIMP1 (50 ng-1 $\mu \mathrm{g}$ ), and MNPs-bound TIMP1 was added (5-20 $\mu \mathrm{g}$ of MNPs loaded with $5 \mu \mathrm{g}$ of TIMP1). After 48 hours of incubation, 6-well plates were given a media change with $1 \mathrm{~mL}$ medium and approximately $100 \mu \mathrm{L}$ of MTT solution (100 mg MTT/20 mL of PBS) was added into each well and incubated at $37^{\circ} \mathrm{C}$ for 3 hours. Finally, $1 \mathrm{~mL}$ of stop solution (20\% sodium dodecyl sulfate in $50 \%$ dimethylformamide) was added into each well and the mixture was kept on a rocker for $\sim 2$ hours; the absorbance was then read at $550 \mathrm{~nm}$ using a microplate reader (Synergy HT Multi-Mode Microplate Reader; BioTek Instrument, Inc.).

\section{Effect of TIMPI on neuronal cell protection from oxidative stress induced by HIV-I infection}

Production of reactive oxygen species (ROS) in HIV-1 infection and/or after adding free TIMP1 or MNP-bound TIMP1 to SK-N-MC cells was detected by the dichlorofluorescein diacetate assay (DCFDA) using the established protocol ${ }^{47}$ with few modifications. SK-N-MC cells were cultured in six-well plates $\left(1 \times 10^{5}\right.$ cells $)$ overnight to allow them to adhere on the bottom surface of the plate. The next day, after activation with polybrene, cells were infected with optimized concentration of HIV-1 for 5 days. On the fifth day, TIMP1 or MNP + TIMP1 NF was added to these cells and incubated for 48 hours. After 7 days of infection/treatment, cells $(100,000$ cells per well) were transferred to 96 -well plates along with its media and incubated overnight. The next day, negative control group wells were treated with catalase $(0.001 \mathrm{mg})$ for 2 hours. Further, cells were treated with $100 \mathrm{mM}$ DCFDA for 1 hour at $37^{\circ} \mathrm{C}$, and finally, the optical density was read in a microplate reader with excitation wavelength of $485 \mathrm{~nm}$ and emission wavelength of $528 \mathrm{~nm}$ (BioTek Instrument, Inc.). Cells treated with $\mathrm{H}_{2} \mathrm{O}_{2}$ for 2 hours were considered as positive control. Untreated and uninfected cells were considered as control cells. Test group results were compared with the control group values.

\section{Spine density measurement}

Coverslips were sterilized using absolute alcohol and placed in six-well culture plates and incubated for 2 hours at $37^{\circ} \mathrm{C}$. After incubation, SK-N-MC cells were cultured on the coverslips and incubated overnight at $37^{\circ} \mathrm{C}$. SK-N-MC cells were treated with the polybrene for 6 hours and infected with the HIV (as explained in the "HIV-1 infection of human SK-N-MC cells" section) for 5 days. On the fifth day of infection, free TIMP1/ MNP-bound TIMP1 was added into the respective wells and incubated for 48 hours. Seven days postinfection, SK-N-MC cells were stained with the Dil (1,1'-dioctadecyl-3,3,3',3'tetramethylindocarbocyanine perchlorate) stain.

\section{Dil stain}

SK-N-MC cells were washed with PBS once and fixed with $4 \%$ formaldehyde for 30 minutes at RT. Then, cells were stained with Dil stain (the fluorescent membrane tracer) $(10 \mu \mathrm{g} / \mathrm{mL}$ in PBS concentration) and incubated for 90 minutes at RT. To allow the transport of the dye, the stained coverslips in six-well plates were incubated overnight in PBS. In the morning, these stained coverslips were mounted on the glass slides with ProLong ${ }^{\circledR}$ Gold Antifade Reagent (Thermo Fisher Scientific, Waltham, MA, USA) and confocal microscopy was performed.

\section{Confocal microscopy}

The stained neuronal cells were observed for dendrites using the TCS SP2 Leica Confocal Laser Scanning Microscope using the $60 \times$ oil immersion objectives, an $88 \mathrm{~nm}$ argonion laser illumination, and a $2.5 \times$ confocal electronic zoom setting. A series of optical serial sections of the cells were used to obtain the best "two-dimensional" images of individual cells. 


\section{Statistical analysis}

All the experiments were performed in duplicate and repeated three times. The obtained values were averaged and data represented as mean \pm standard error (SE). GraphPad Prism software was used to analyze the data. One-way analysis of variance was used to compare the data obtained between the groups, and the results were considered significant at $P \leq 0.05$. The confocal images were analyzed for the spine density using the ImageJ software.

\section{Results}

\section{Formulation characterization and drug release kinetics}

MNPs were prepared per laboratory standardized protocol $^{43}$ and characterized for size, shape, polydispersity index, and TIMP1 loading. TEM studies show that nanoparticles were in the range of $10 \pm 3 \mathrm{~nm}$ (TIMP1 loaded), as shown in Figure 1A, and had excellent dispersion property (polydispersity index $>0.178$ ) in aqueous medium.

The synthesized MNPs were also analyzed for superparamagnetic property (magnetic hysteresis loops, range: $+1,200$ to $-1,200$ Oersted) and crystal structure (X-ray diffraction spectroscopy); the results showed strong magnetic behavior (revealed through no coercivity and remanence) and magnetitespecific peaks (data not shown). Also, the time kinetics of TIMP1 $(5 \mu \mathrm{g})$ loading with respect to MNP concentration was evaluated - maximum binding of TIMP1 $(4 \pm 0.51 \mu \mathrm{g}$, corresponds to $80 \%$ loading) occurred as early as 10 minutes when lowest amount of MNPs (5 $\mu \mathrm{g})$ was used as shown in Figure 1B ( $a$ and b), compared to higher time of incubation or larger amount of MNPs. Zeta $(\zeta)$ potential showed overall negative charge on MNPs $(-30 \pm 4 \mathrm{mV})$ in deionized water, which changed to $+10 \pm 2 \mathrm{mV}$ on TIMP1 loading, thus confirming the TIMP1 deposition on MNPs due to electrostatic bonding. Furthermore, in vitro pharmacokinetic release studies showed that $100 \%$ TIMP1 release from MNPs takes place in $\sim 6$ hours in $\mathrm{PBS}(\mathrm{pH} 7.4)$ at $37^{\circ} \mathrm{C}$ (Figure 1C).

\section{In vitro BBB development and MNP + TIMPI NF transmigration efficiency studies}

The BBB transmigrability of the plain MNPs and MNPs + TIMP1 NF was evaluated using an in vitro human BBB model. The tightness of the developed in vitro $\mathrm{BBB}$ was
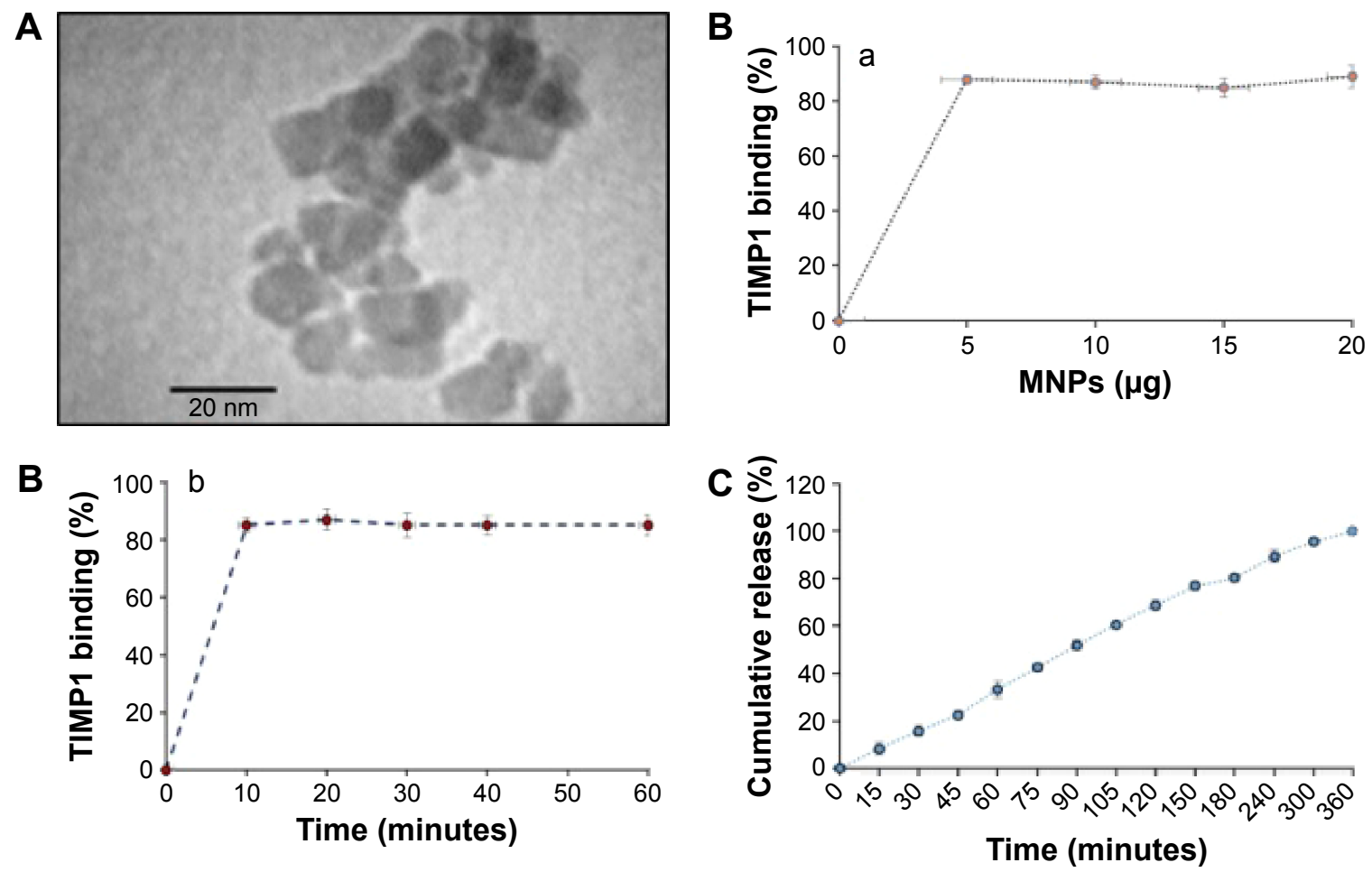

Figure I Characterizations of MNP nanoformulation.

Notes: (A) Transmission electron microscopy of TIMPI-loaded MNPs; (B) binding kinetics of TIMPI (5 $\mu$ g) to MNPs: (a) effect of MNPs amount with respect to percentage TIMPI binding and (b) effect of time with respect to percentage TIMPI binding; (C) in vitro release data: cumulative percentage release profile of TIMPI from MNP nanoformulation (5 $\mu \mathrm{g}$ of TIMPI loaded onto $5 \mu \mathrm{g}$ MNPs) was studied in PBS (pH 7.4) with $0.1 \%$ sodium azide at $37^{\circ} \mathrm{C}$ using $100 \mathrm{kD}$ Micro Float-A-Lyzer (Spectrum Labs, Cincinnati, OH, USA) dialysis membrane. Standard errors are shown for the mean of triplicate samples.

Abbreviations: TIMPI, tissue inhibitor of metalloproteinase-I; MNPs, magnetic nanoparticles; PBS, phosphate-buffered saline. 
Table I In vitro BBB model TEER values

\begin{tabular}{llll}
\hline Magnetic force & $\begin{array}{l}\text { Untreated } \\
\text { well (control) }\end{array}$ & $\begin{array}{l}\text { Plain } \\
\text { MNPs }\end{array}$ & TIMPI + MNPs \\
\hline No force & $362.5 \pm 9.7$ & $371.8 \pm 5.6$ & $360.6 \pm 9.5$ \\
Magnetic force & $362.6 \pm 6.5$ & $368.4 \pm 10.5$ & $360.2 \pm 2.6$
\end{tabular}

Note: Data presented as mean \pm standard deviation. $n=5$.

Abbreviations: BBB, blood-brain barrier; MNPs, magnetic nanoparticles; TEER, transendothelial electrical resistance; TIMPI, tissue inhibitor of metalloproteinase-I.

determined by TEER values estimation after 5-6 days as per our previously standardized protocol. ${ }^{43}$ Following the TEER determination, $100 \mu \mathrm{L}$ of cell culture medium containing MNPs $(5 \mu \mathrm{g}) \pm$ TIMP1 $(4 \pm 0.51 \mu \mathrm{g}) \mathrm{NF}$ was added in the upper chamber into the respective wells (24-well plate) and incubated in the presence or absence of external magnetic force $(0.8 \mathrm{~T})$. As shown in Table 1 , before applying the magnetic force, TEER values of all treatment groups were comparable to the standard (untreated well) of $360 \pm 10 \Omega / \mathrm{cm}^{2}$. As per our expectation, even after applying the magnetic force, we did not observe any significant change in TEER value with MNPs + TIMP1 NF treatment (as shown in Table 1) when compared with untreated and plain MNP samples.

MNPs + TIMP1 NF ability with respect to BBB transmigration was verified by measuring the iron concentration in the lower chamber after application of external magnetic field for different time points. We observed that $40 \% \pm 2.5 \%$ of plain MNPs and $37.5 \% \pm 1.5 \%$ of MNPs + TIMP1 NF transmigrated across the in vitro BBB on magnetic treatment (0.8 T, 2 hours) compared to no magnetic treatment for both MNPs and MNPs + TIMP1 NF (shown in Figure 2A). Also, no significant increase in nanoformulation transmigration
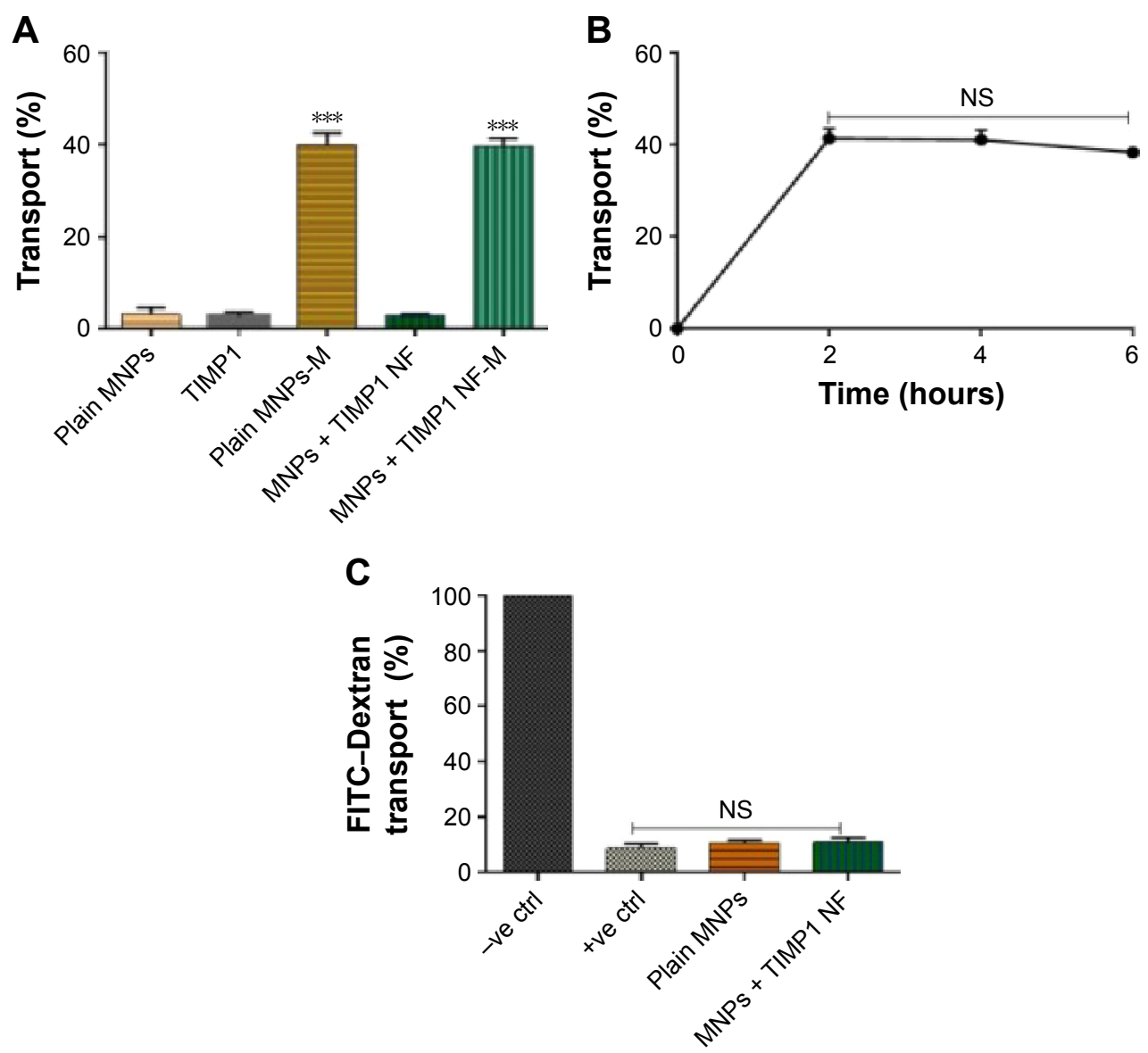

Figure 2 MNPs + TIMPI nanoformulation (NF) transmigration study using in vitro BBB model.

Notes: (A) Transmigration efficiency evaluation: MNPs, free TIMPI, and MNPs + TIMPI NF were added in the upper chamber of the transwells, and samples were tested for transmigration ability in the presence or absence of static magnet $(0.8 \mathrm{~T})$ placed underneath the cell culture plate for the 2 hours treatment; transmigrated samples were collected from the bottom chamber, centrifuged, and estimated for iron content using iron assay; (B) effect of magnetic treatment time with respect to NF transmigration efficiency: samples from the lower chamber at different time points, ie, 2, 4, and 6 hours, were collected, and transmigrated MNPs + TIMPI NF was collected from the bottom chamber, centrifuged, and calculated for iron content using iron assay. (C) BBB integrity analysis using the FITC-dextran transport assay: FITC-dextran transport was measured for MNPs/MNPs + TIMPI NF after 2 hours' magnetic treatment, and FITC-dextran (molecular weight 40,000 Da) was added to the upper chamber of the insert. After 30 minutes' incubation, relative fluorescence unit from the lower chamber of the transwell was measured. Results were expressed as \% FITC-dextran transport with respect to the untreated control cultures (-ve control $=$ no cell and +ve control $=$ with all BBB cells) and represented as mean $\pm S E$ of independent experiments. *** $P \leq 0.00 \mathrm{I}$.

Abbreviations: BBB, blood-brain barrier; SE, standard error; FITC, fluorescein isothiocyanate; TIMPI, tissue inhibitor of metalloproteinase-I; NF, nanoformulation; MNPs, magnetic nanoparticles; NS, not significant; ctrl, control; M, Magnet. 
was observed on applying longer period of magnetic field as shown in Figure 2B.

Further, the integrity/intactness of BBB was evaluated using paracellular transport of FITC-dextran as described earlier in the methodology, with FITC molecule used as the detection moiety to confirm the membrane intactness. As can be seen from Figure $2 \mathrm{C}$, only $7.25 \% \pm 3 \%$ FITC is transported (upper to lower chamber) for both MNPs and MNPs + TIMP1 $\mathrm{NF}$ in comparison to $\sim 100 \%$ transport for negative control (transwell without any BBB cells), thus proving that MNPs + TIMP1 NF transmigration under external magnetic field does not affect the BBB integrity and that the nanoformulation is safe to be used for clinical application.

\section{In vitro cytotoxicity and cell uptake studies}

To explore the effect of different amounts of free TIMP1, MNPs, and MNP-tagged TIMP1 on SK-N-MC cells infected with HIV, the MTT assay was used. We did not observe any significant cytotoxicity in HIV-infected SK-N-MC cells exposed to free TIMP1 (up to $1 \mu \mathrm{g}$ ), MNPs (up to $20 \mu \mathrm{g}$ ), and MNP-tagged TIMP1 (5 $\mu \mathrm{g}$ MNPs $+5 \mu \mathrm{g}$ TIMP1) combination when compared to the control uninfected and untreated SK-N-MC cells (Figure 3), thus suggesting NF is nontoxic

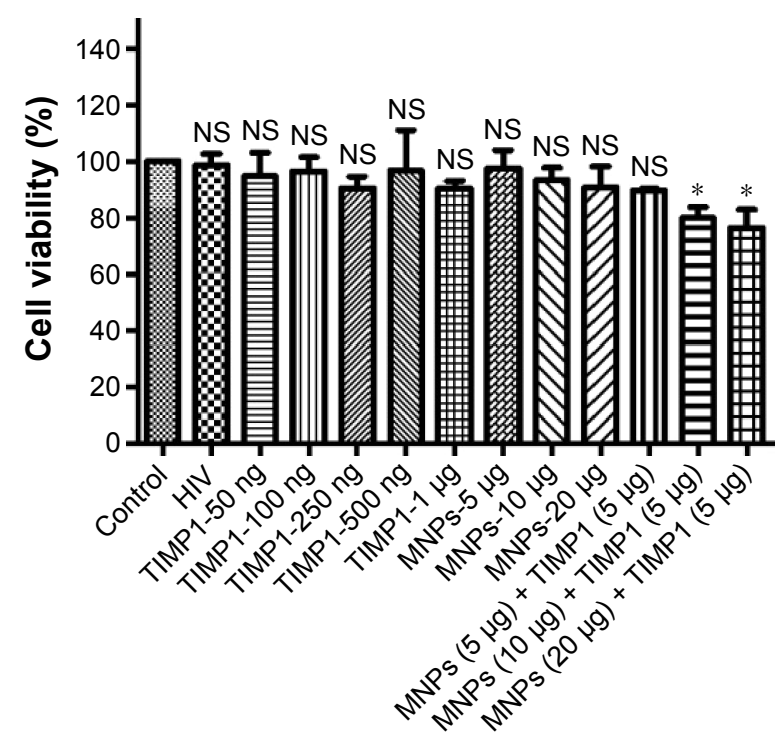

Figure 3 Cytotoxicity studies in SK-N-MC cells for free and TIMPI + MNPs nanoformulation components.

Notes: Figure shows the percentage of cells viable after treatment with different amounts of MNPs $(5-20 \mu \mathrm{g} / \mathrm{mL})$ or free TIMPI $(50 \mathrm{ng}-\mathrm{I} \mu \mathrm{g})$ or MNPs + TIMPI nanoformulation. SK-N-MC cells $\left(I \times 10^{5}\right.$ cells) were grown in the six-well culture plates, and cells were infected with $20 \mathrm{ng}$ of HIV-I clade B overnight. Unbound virus was washed, and cells were infected for 7 days. On fifth day of infection, TIMPI/ MNPs/MNPs + TIMPI was added at different amounts into the respective wells and incubated for 48 hours. Seven days postinfection, cell viability was measured using the MTT assay. $* P \leq 0.05$.

Abbreviations: HIV, human immunodeficiency virus; TIMPI, tissue inhibitor of metalloproteinase-I; NF, nanoformulation; MNPs, magnetic nanoparticles; NS, not significant. in nature and should not have any safety concerns for any preclinical use.

Also, the quantitative analysis of MNPs + TIMP1 NF for cell uptake was performed by using the flow-cytometry. Our results showed that maximum cell uptake took place as early as 3 hours $(\sim 50 \%)$ and that there was no significant difference in cell uptake (percentage of cells positive for FITC-tagged NF) with longer incubation time, ie, up to 24 hours (as shown in Figure 4).

\section{Decreased HIV-I infection in SK-N-MC neuronal cells exposed to MNP-bound TIMPI}

SK-N-MC cells were infected with HIV for 5 days, and free TIMP1 alone or MNP-tagged TIMP1 was added to the infected cells and incubated further for 48 hours. After a total of 7 days of infection, supernatant was collected and HIV infectivity in these cells was measured using the p24 ELISA. Although we did not expect any difference in HIV infection with TIMP1 treatment, surprisingly, we observed significant reduction of HIV infection in TIMP1-treated (starting from 250 ng of TIMP1) and MNPs + TIMP1 NF-treated SK-N-MC cells (Figure 5).

\section{Reduced oxidative stress in HIV-infected SK-N-MC neuronal cells exposed to free TIMPI or MNP-bound TIMPI}

HIV infection is known to induce oxidative stress by production of ROS in different types of cells. To see the effect of HIV infection on ROS production in SK-N-MC cells and to investigate the neuroprotective role of TIMP1, we analyzed the ROS production in HIV-infected cells exposed to the solution-phase free TIMP1 and MNPs + TIMP1 NF. We observed significant reduction of ROS production in HIV-infected SK-N-MC cells exposed to solution-phase free TIMP1 when compared to the cells infected with HIV alone. In case of MNPs + TIMP1 NF exposed HIV-infected SK-N-MC cells, we observed significantly reduced ROS production when compared to the cells infected with HIV alone. We did not find significant difference in ROS production between HIV-infected cells exposed to free TIMP1 and MNPs + TIMP1 NF (Figure 6).

\section{TIMPI recovers spine density in HIV-infected neurons}

In the HIV-infected SK-N-MC cells, we observed significantly reduced spine density when compared to the uninfected control cells. When these cells were exposed to free TIMP1 alone 


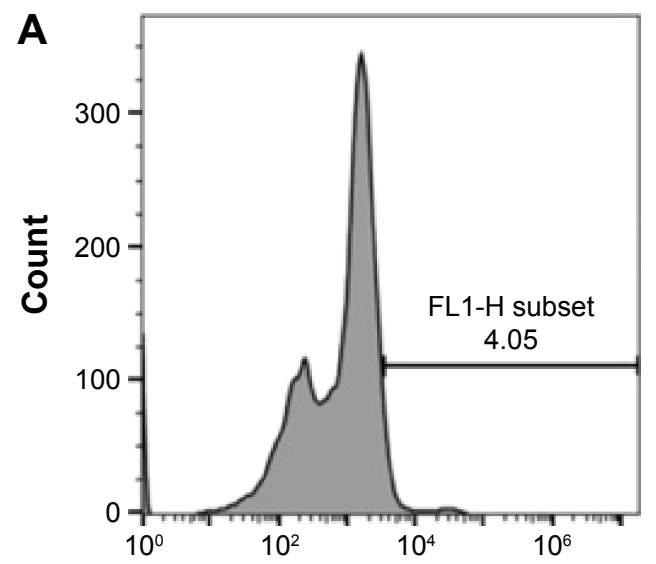

FL1-H::FL1-H

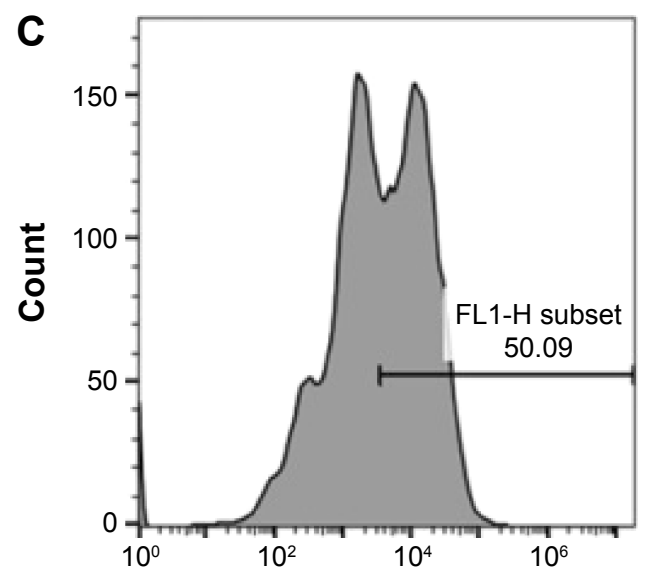

FL1-H::FL1-H

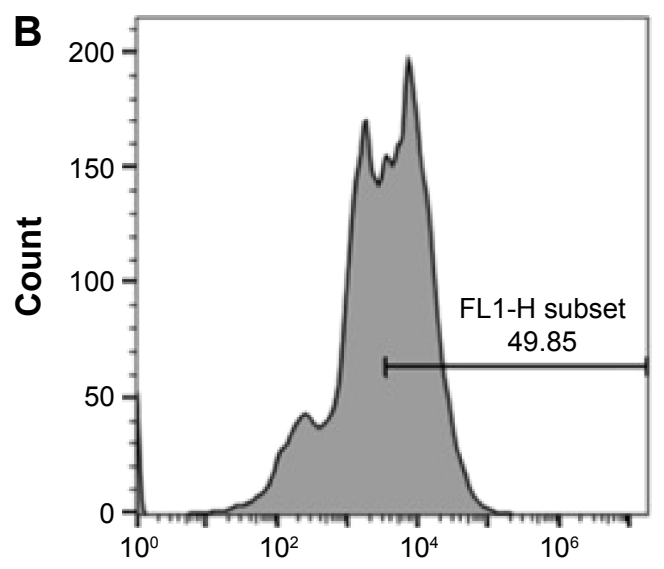

FL1-H::FL1-H

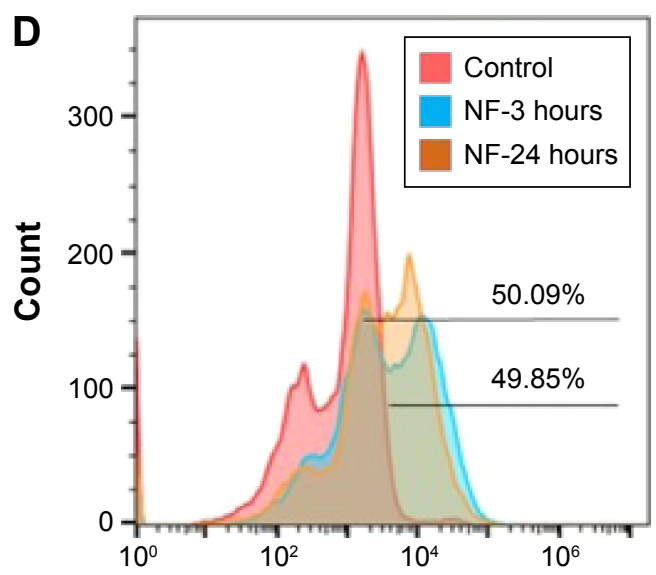

FL1-H::FL1-H

Figure 4 Quantitative analysis of uptake of FITC-tagged MNPs + TIMPI NF in SK-N-MC cells using the flow cytometry.

Notes: SK-N-MC cells were exposed to $5 \mu \mathrm{g}$ of FITC-labeled MNPs + TIMPI NF for 3, 6, I2, and 24 hours. Uptake of the NF was measured by the fluorescence in the SK-N-MC cells using the flow cytometry. For each sample, 10,000 events were collected, and cells were gated based on the cells without NF (pink-line histogram) or control (A). Colored histograms show cells positive for NF with shifted mean fluorescence intensity compared to controls. The representative figures for uptake at 3 hours (B) and 24 hours (C) are presented. Histograms represent cells treated with $5 \mu \mathrm{g}$ of FITC-tagged NF for 3 hours (blue) and 24 hours (orange) (D), which show $49.85 \%$ and $50 \%$ cells are positive for MNPs + TIMPI NF, respectively, when compared with the untreated cells. Histograms show an overlay for time points (3 and 24 hours) (D).

Abbreviations: FITC, fluorescein isothiocyanate; TIMPI, tissue inhibitor of metalloproteinase-I; NF, nanoformulation; MNPs, magnetic nanoparticles.

or MNP-bound TIMP1, we observed significant recovery of spine density when compared to the HIV-infected cells. We did not find significant difference in the recovery of the spine density between free TIMP1-treated cells and MNP-bound TIMP1-treated cells (Figure 7).

\section{Discussion}

Neuronal cell death or apoptosis by means of oxidative stress in HIV-infected patients is one of the key mechanisms responsible for the cognitive and motor dysfunctions. ${ }^{48,49}$ In our previous studies, we have observed dysregulation of synaptic plasticity genes and decreased spine density in HIV-infected neuronal cells. ${ }^{6}$ Therefore, there is a need to investigate the therapeutic molecules that can induce neuroprotection in HIV-infected patients. In vitro and in vivo models of brain trauma and stroke suggest that TIMP1 is neuroprotective and that overexpression of TIMP1 prevents the BBB leakage and reduces neuronal cell death. ${ }^{50}$ Downregulation of TIMP1 was observed in HIV-infected macrophages and vascular endothelial cells. ${ }^{51}$ In our previous studies, among different dysregulated genes, TIMP1 was one of the highly downregulated synaptic plasticity genes in HIV-infected neuronal cells. ${ }^{6}$ In addition to our in vitro reports, in chronically HIV-infected patients, decreased TIMP1 protein expression was observed in brain tissue and cerebrospinal fluid. ${ }^{52}$ TIMP1 is not only the inhibitor of MMPs-1-3, -7-13, and -16 but also acts as a candidate synaptic plasticity gene (induces expression of immediate early response genes) leading to the development of long-term potentiation, which plays a major role in forming learning and memory. ${ }^{53-55}$ TIMP1 has been reported to have neuroprotective effects equivalent to that of brain-derived neurotropic factor. ${ }^{50}$ Therefore, supplementing TIMP1 may 


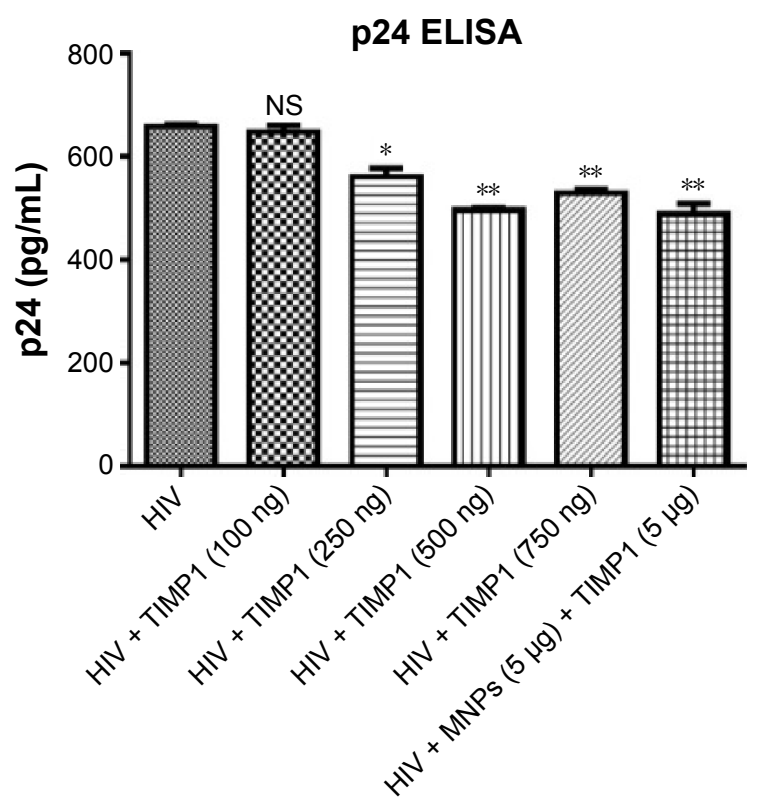

Figure 5 Decreased HIV infection levels in SK-N-MC cells treated with free TIMPI or MNPs + TIMPI NF.

Notes: SK-N-MC ( $1 \times 10^{5}$ cells) were grown in the six-well culture plates, and cells were infected with $20 \mathrm{ng}$ of HIV-I clade B overnight. Unbound virus was washed with PBS, and cells were infected for 7 days. On fifth day of infection, free TIMPI/ MNPs + TIMPI NF was added into the respective wells and incubated for 48 hours. Seven days postinfection, HIV infection levels were measured using the p24 ELISA. $* P \leq 0.05, * * P \leq 0.01$

Abbreviations: TIMPI, tissue inhibitor of metalloproteinase-I; NF, nanoformulation; MNPs, magnetic nanoparticles; HIV, human immunodeficiency virus; PBS, phosphatebuffered saline; ELISA, enzyme-linked immunosorbent assay; NS, not significant.

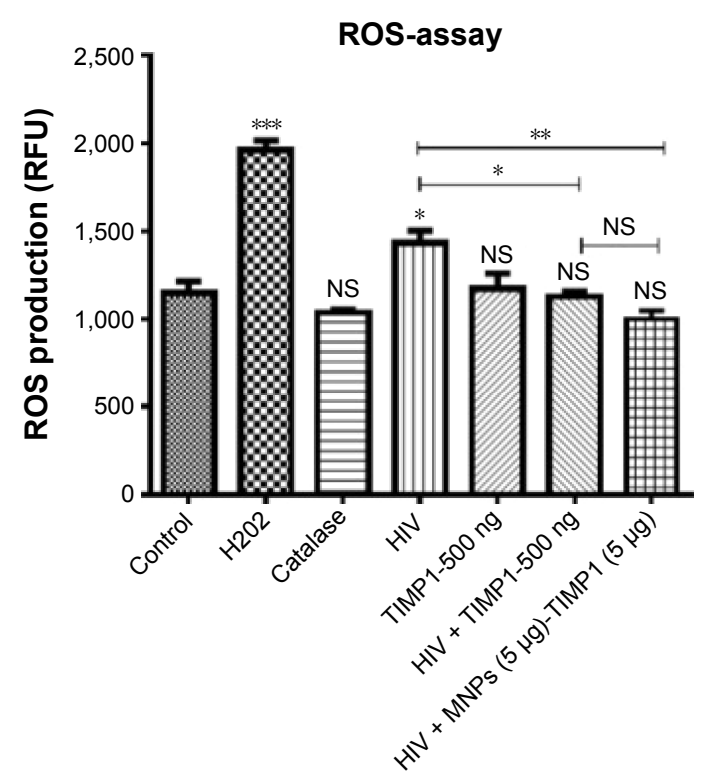

Figure 6 Decreased ROS production in HIV-infected SK-N-MC cells treated with free TIMPI or MNPs + TIMPI NF.

Notes: SK-N-MC $\left(I \times 10^{5}\right.$ cells) were grown in the six-well culture plates, and cells were infected with $20 \mathrm{ng}$ of HIV-I clade B overnight. Unbound virus was washed with phosphate-buffered saline, and cells were infected for 7 days. On fifth day of infection, TIMPI/MNPs/MNPs + TIMPI NF was added into the respective wells and incubated for 48 hours. Seven days postinfection, ROS production was measured using the ROS assay. $* P \leq 0.05, * * P \leq 0.01$, ***P $\leq 0.00$ I.

Abbreviations: ROS, reactive oxygen species; RFU, relative fluorescence unit; TIMPI, tissue inhibitor of metalloproteinase-I; NF, nanoformulation; MNPs, magnetic nanoparticles; HIV, human immunodeficiency virus; NS, not significant. be helpful in reducing the neuronal damage induced by the HIV infection. Due to the selectively permeable nature of the BBB, novel and effective delivery methods are needed for the successful delivery of therapeutic molecules across the BBB. In this study, we used MNPs for the delivery of TIMP1 protein across the $\mathrm{BBB}$ for neutralizing the neuropathogenesis induced in HIV infection. We observed successful delivery of TIMP1 nanoformulation ( 40\%) across in vitro BBB model on application of external magnetic field. Also, results showed no damage to BBB integrity after transmigration of NF as shown in Figure 2. The percentage of transmigration of free MNPs or MNP-bound TIMP1 NF transmigration across the $\mathrm{BBB}$ is in the range of our previous studies. ${ }^{43,56}$

We also did not observe neuronal cytotoxicity of the free TIMP1 (up to $1 \mu \mathrm{g}$ ), free MNPs (up to $20 \mu \mathrm{g}$ ), and MNPbound TIPM1 NF. In this study, surprisingly, we observed significantly reduced HIV infection in SK-N-MC cells in the presence of free TIMP1 and MNP-bound TIMP1 NF when compared with HIV controls. But we did not see any significant difference in the HIV infection between the group of SK-N-MC cells grown in the presence of free TIMP1 and MNP-bound TIMP1 protein. Although TIMP1 controls the activity of MMP-1-3, -7-13, and -16, it has the strongest affinity for the MMP-2 and -9. In HIV-infected patients, increased secretion of MMP-2 and MMP-9 induces extracellular matrix degradation, which leads to the disruption of endothelial barriers, thus facilitating the spread of HIV infection in tissues. ${ }^{57}$ Reduced TIMP1 expression over time has been reported to potentially contribute to an increased propensity of HIV infection within the brain parenchyma. ${ }^{58}$ Based on our results and previous reports, it is indicative that supplementation of soluble-phase TIMP1 or MNP-bound TIMP1 results in the reduced infectivity levels in the neuronal cells and that this may be due to the inhibition of MMP-2 and MMP-9 activity. In a similar fashion, in glial cells and peripheral blood mononuclear cells, antiretroviral therapy was reported to inhibit the expression of MMP, indicating that the beneficial effects of antiretroviral therapy in HIV-infected patients may also be due to the reduction of MMP levels. ${ }^{57,59}$ Therefore, mechanisms for reducing HIV infectivity in the presence of TIMP1 need to be further investigated in various types of peripheral and CNS cells.

There are many strong evidences that support the fact that various materials at the nanometer range have the ability to induce the production of ROS. ${ }^{60}$ Hence, we wanted to determine whether our NFs had any effects on the production of ROS as it involved iron oxide nanoparticle as a carrier. We did not observe any cytotoxicity/oxidative stress with the amount of MNPs that we used in our studies. This is in 

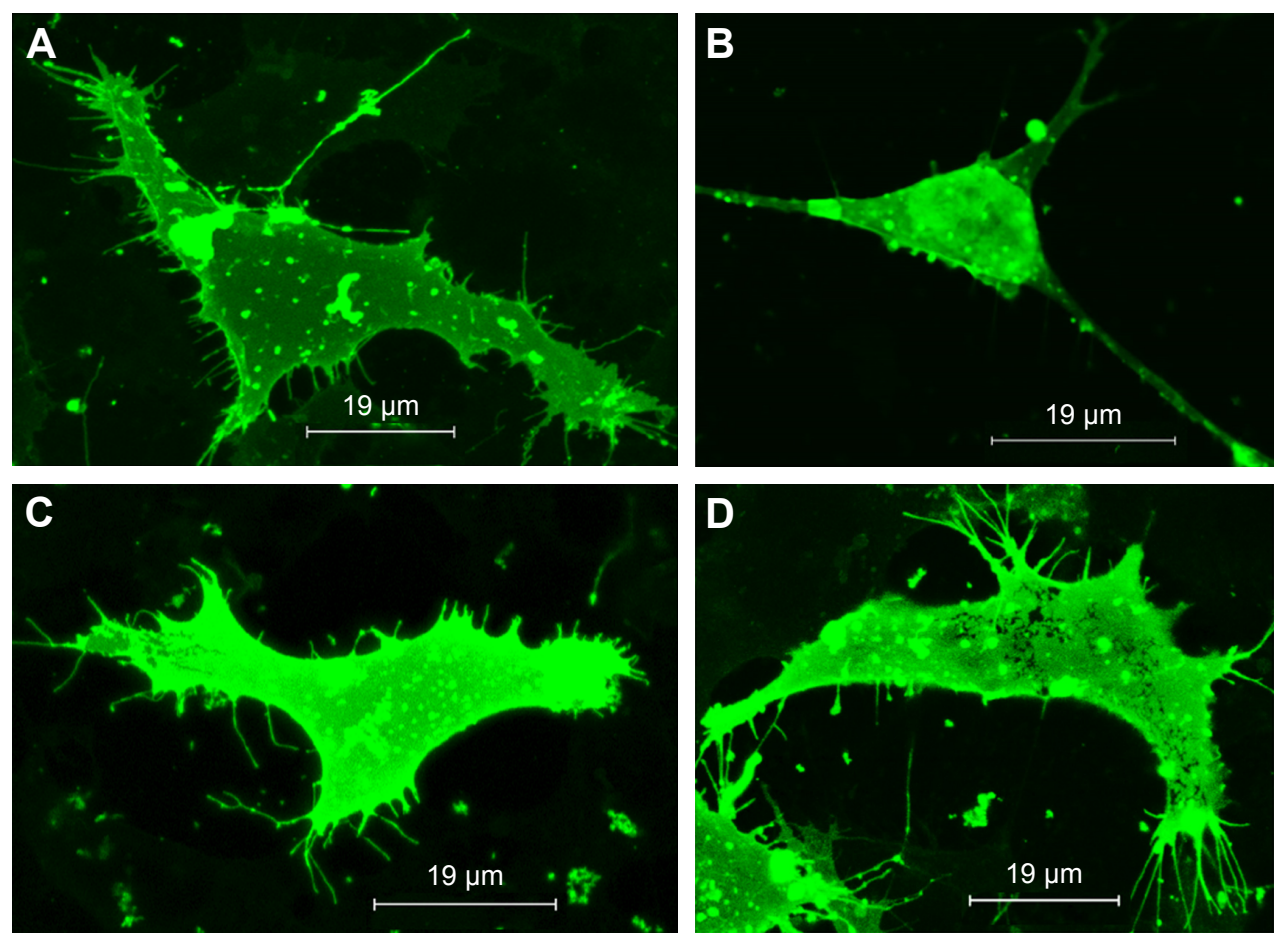

Figure 7 TIMPI recovers spine density in human immunodeficiency virus (HIV)-infected neuronal cells.

Notes: SK-N-MC cells were infected with HIV for 7 days. On the fifth day of HIV infection, free TIMPI or MNPs + TIMPI nanoformulation was added to the culture. (A) Control cells: long spines in high number can be observed on a dendritic length, (B) HIV-infected cells: loss of number of spines can be observed on a dendritic length when compared to the control. (C) HIV-infected cells in the presence of free TIMPI: recovery of spines on the dendrite length when compared to the HIV-infected cells. (D) HIV-infected cells in the presence of MNPs + TIMPI NF: recovery of spines on the dendrite length when compared to the HIV-infected cells.

Abbreviations: TIMPI, tissue inhibitor of metalloproteinase-I; MNPs, magnetic nanoparticles; HIV, human immunodeficiency virus; NF, nanoformulation.

agreement with the previous reports that cytotoxicity and oxidative stress induction by MNPs in the cells are dose dependent. ${ }^{61}$ Furthermore, in the presence of both solutionphase and MNP-bound TIMP1, we observed significantly reduced oxidative stress induced by HIV infection. Several reports indicate that oxidative stress plays a major role in neuronal damage and cognitive disorders in HIV-infected patients. ${ }^{62,63}$ In this study, we observed that reduced oxidative stress could be due to the decreased HIV infection because of release of TIMP1 from the MNP NF. We also observed the effect of free TIMP1 and MNP-bound TIMP1 exposure on the recovery of spine density in HIV-infected neuronal cells. In both the test groups, we observed recovery of spine density compared to the HIV-infected cells. The recovery of spine density may also be due to the decreased HIV infection, reduced oxidative stress, and induced synaptic plasticity genes expression. Also, the recovery of spine density plays a major role in the generation of long-term potentiation and formation of long-term memory. Therefore, recovery of spine density may help in the recovery of synaptic plasticity and reduce the neurocognitive impairments observed in the HIV-infected patients. Therefore, based on these exciting and novel findings, in future, we would like to test the efficacy of the developed NF in vivo to take it to the clinical level for neuroprotection in HIV-infected patients.

\section{Conclusion}

Our results indicate that MNP-bound TIMP1 NF may act as a neuroprotective agent by decreasing the HIV infection levels and oxidative stress, thus enabling the recovery of neuronal spine density. Due to the impermeability of the BBB, the use of MNP-bound TIMP1 helps in successful delivery of the TIMP1 protein across the BBB into the neuronal cells. In conclusion, these results highlight a novel function, mechanism, and direct role of TIMP1 as a neuroprotective agent in HIV-induced neurotoxicity, signifying MNPs + TIMP1 NF delivery as a therapy for neurodegenerative diseases.

\section{Acknowledgments}

The authors acknowledge financial support from NIH grants R01DA027049, R01DA037838, R01MH085259, R01DA040537, and R01DA034547.

\section{Author contributions}

Atluri and Jayant conducted all the key measurements including MNP synthesis, TIMP1 nanoformulation preparation, 
in vitro pharmacokinetic release studies, BBB preparation, validation and release across $\mathrm{BBB}$, and confocal microscopy for spine density studies. Garcia helped in cell culture studies. Kaushik helped in transmission electron microscopy and X-ray diffraction studies. Samikkannu and Kanthikeel helped in designing cellular uptake studies and its analysis. Adriana helped in flow cytometer sample preparation and data analysis. Nair oversaw and supervised the entire project. All authors contributed toward data analysis, drafting and critically revising the paper and agree to be accountable for all aspects of the work.

\section{Disclosure}

The authors report no conflicts of interest in this work.

\section{References}

1. Moroni M, Antinori S. HIV and direct damage of organs: disease spectrum before and during the highly active antiretroviral therapy era. AIDS. 2003;17(Suppl 1):S51-S64.

2. Atluri VS, Kanthikeel SP, Reddy PV, Yndart A, Nair MP. Human synaptic plasticity gene expression profile and dendritic spine density changes in HIV-infected human CNS cells: role in HIV-associated neurocognitive disorders (HAND). PLoS One. 2013;8(4):e61399.

3. Gonzalez-Scarano F, Martin-Garcia J. The neuropathogenesis of AIDS Nat Rev Immunol. 2005;5(1):69-81.

4. Alvarez Losada S, Canto-Nogues C, Munoz-Fernandez MA. A new possible mechanism of human immunodeficiency virus type 1 infection of neural cells. Neurobiol Dis. 2002;11(3):469-478.

5. Harouse JM, Collman RG, Gonzalez-Scarano F. Human immunodeficiency virus type 1 infection of SK-N-MC cells: domains of gp120 involved in entry into a CD4-negative, galactosyl ceramide/3 sulfo-galactosyl ceramide-positive cell line. J Virol. 1995;69(12): 7383-7390.

6. Atluri VS, Pilakka-Kanthikeel S, Samikkannu T, et al. Vorinostat positively regulates synaptic plasticity genes expression and spine density in HIV infected neurons: role of nicotine in progression of HIV-associated neurocognitive disorder. Mol Brain. 2014;7:37.

7. Masliah E, DeTeresa RM, Mallory ME, Hansen LA. Changes in pathological findings at autopsy in AIDS cases for the last 15 years. AIDS 2000;14(1):69-74.

8. Gray F, Adle-Biassette H, Chretien F, Lorin de la Grandmaison G, Force G, Keohane C. Neuropathology and neurodegeneration in human immunodeficiency virus infection. Pathogenesis of HIV-induced lesions of the brain, correlations with HIV-associated disorders and modifications according to treatments. Clin Neuropathol. 2001;20(4): 146-155.

9. Aylward EH, Henderer JD, McArthur JC, et al. Reduced basal ganglia volume in HIV-1-associated dementia: results from quantitative neuroimaging. Neurology. 1993;43(10):2099-2104.

10. Berger JR, Arendt G. HIV dementia: the role of the basal ganglia and dopaminergic systems. J Psychopharmacol. 2000;14(3):214-221.

11. Stamenkovic I. Extracellular matrix remodelling: the role of matrix metalloproteinases. J Pathol. 2003;200(4):448-464.

12. Murphy G, Nagase H. Progress in matrix metalloproteinase research. Mol Aspects Med. 2008;29(5):290-308.

13. Gill SE, Parks WC. Metalloproteinases and their inhibitors: regulators of wound healing. Int J Biochem Cell Biol. 2008;40(6-7): 1334-1347.

14. Radisky ES, Radisky DC. Matrix metalloproteinase-induced epithelialmesenchymal transition in breast cancer. J Mammary Gland Biol Neoplasia. 2010;15(2):201-212.
15. Brew K, Dinakarpandian D, Nagase H. Tissue inhibitors of metalloproteinases: evolution, structure and function. Biochim Biophys Acta. 2000; 1477(1-2):267-283.

16. Murphy G. Tissue inhibitors of metalloproteinases. Genome Biol. 2011; 12(11):233.

17. Berman NEJ, Marcario JK, Yong C, et al. Microglial activation and neurological symptoms in the SIV model of NeuroAIDS: association of MHC-II and MMP-9 expression with behavioral deficits and evoked potential changes. Neurobiol Dis. 1999;6(6):486-498.

18. Lorenzl S, Albers DS, Relkin N, et al. Increased plasma levels of matrix metalloproteinase-9 in patients with Alzheimer's disease. Neurochem Int. 2003;43(3):191-196.

19. Muroski ME, Roycik MD, Newcomer RG, et al. Matrix metalloproteinase-9/gelatinase $B$ is a putative therapeutic target of chronic obstructive pulmonary disease and multiple sclerosis. Curr Pharm Biotechnol. 2008;9(1):34-46.

20. BÉLiveau R, Delbecchi L, Beaulieu E, et al. Expression of matrix metalloproteinases and their inhibitors in human brain tumors. Ann $N$ Y Acad Sci. 1999;886(1):236-239.

21. Yin P, Yang L, Zhou HY, Sun RP. Matrix metalloproteinase-9 may be a potential therapeutic target in epilepsy. Med Hypotheses. 2011; 76(2):184-186.

22. Jourquin J, Tremblay E, Décanis N, et al. Neuronal activity-dependent increase of net matrix metalloproteinase activity is associated with MMP-9 neurotoxicity after kainate. Eur J Neurosci. 2003;18(6): $1507-1517$.

23. Coussens LM, Fingleton B, Matrisian LM. Matrix metalloproteinase inhibitors and cancer: trials and tribulations. Science. 2002;295(5564): 2387-2392.

24. Turk B. Targeting proteases: successes, failures and future prospects. Nat Rev Drug Discov. 2006;5(9):785-799.

25. Burrage PS, Brinckerhoff CE. Molecular targets in osteoarthritis: metalloproteinases and their inhibitors. Curr Drug Targets. 2007;8(2): 293-303.

26. Fingleton B. MMPs as therapeutic targets - still a viable option? Semin Cell Dev Biol. 2008;19(1):61-68.

27. Van den Steen PE, Dubois B, Nelissen I, Rudd PM, Dwek RA, Opdenakker G. Biochemistry and molecular biology of gelatinase B or matrix metalloproteinase-9 (MMP-9). Crit Rev Biochem Mol Biol. 2002; 37(6):375-536.

28. Ashutosh Chao C, Borgmann K, Brew K, Ghorpade A. Tissue inhibitor of metalloproteinases-1 protects human neurons from staurosporine and HIV-1-induced apoptosis: mechanisms and relevance to HIV-1associated dementia. Cell Death Dis. 2012;3(6):e332.

29. Chaturvedi M, Molino Y, Sreedhar B, Khrestchatisky M, Kaczmarek L. Tissue inhibitor of matrix metalloproteinases-1 loaded poly(lactic-coglycolic acid) nanoparticles for delivery across the blood-brain barrier. Int J Nanomedicine. 2014;9:575-588.

30. Pardridge WM. Blood-brain barrier delivery. Drug Discov Today. 2007;12(1-2):54-61.

31. Mandel RJ, Gage FH, Clevenger DG, Spratt SK, Snyder RO, Leff SE. Nerve growth factor expressed in the medial septum followingin vivogene delivery using a recombinant adeno-associated viral vector protects cholinergic neurons from fimbria-fornix lesion-induced degeneration. Exp Neurol. 1999;155(1):59-64.

32. Blits B, Oudega M, Boer GJ, Bartlett Bunge M, Verhaagen J. Adenoassociated viral vector-mediated neurotrophin gene transfer in the injured adult rat spinal cord improves hind-limb function. Neuroscience. 2003;118(1):271-281.

33. Williams LR, Varon S, Peterson GM, et al. Continuous infusion of nerve growth factor prevents basal forebrain neuronal death after fimbria fornix transection. Proc Natl Acad Sci U S A. 1986;83(23):9231-9235.

34. Thoenen H, Sendtner M. Neurotrophins: from enthusiastic expectations through sobering experiences to rational therapeutic approaches. Nat Neurosci. 2002;5(Suppl):1046-1050.

35. Alexiou C, Schmid RJ, Jurgons R, et al. Targeting cancer cells: magnetic nanoparticles as drug carriers. Eur Biophys J. 2006;35(5):446-450. 
36. Lübbe AS, Bergemann C, Huhnt W, et al. Preclinical experiences with magnetic drug targeting: tolerance and efficacy. Cancer Res. 1996; 56(20):4694-4701.

37. Nair M, Jayant RD, Kaushik A, Sagar V. Getting into the brain: potential of nanotechnology in the management of neuroAIDS. Adv Drug Deliv Rev. 2016. 103:202-217.

38. Jayant R, Nair M. Nanotechnology for the treatment of NeuroAIDS. J Nanomed Res. 2016;3(1):00047.

39. Sagar V, Pilakka-Kanthikeel S, Atluri VS, et al. Therapeutical neurotargeting via magnetic nanocarrier: implications to opiate-induced neuropathogenesis and neuroAIDS. J Biomed Nanotechnol. 2015; 11(10):1722-1733.

40. Ding H, Sagar V, Agudelo M, et al. Enhanced blood-brain barrier transmigration using a novel Transferrin-embedded fluorescent magnetoliposome nanoformulation. Nanotechnology. Epub 2014 Jan 9.

41. Nair M, Guduru R, Liang P, Hong J, Sagar V, Khizroev S. Externally controlled on-demand release of anti-HIV drug using magneto-electric nanoparticles as carriers. Nat Commun. 2013;4:1707.

42. Kaushik A, Jayant RD, Nikkhah-Moshaie R, et al. Magnetically guided central nervous system delivery and toxicity evaluation of magnetoelectric nanocarriers. Sci Rep. 2016;6:25309.

43. Jayant RD, Atluri VS, Agudelo M, Sagar V, Kaushik A, Nair M. Sustained-release nanoART formulation for the treatment of neuroAIDS. Int J Nanomedicine. 2015;10:1077-1093.

44. Persidsky Y, Stins M, Way D, et al. A model for monocyte migration through the blood-brain barrier during HIV-1 encephalitis. J Immunol. 1997;158(7):3499-3510.

45. Toduka Y, Toyooka T, Ibuki Y. Flow cytometric evaluation of nanoparticles using side-scattered light and reactive oxygen species-mediated fluorescence correlation with genotoxicity. Environ Sci Technol. 2012;46(14):7629-7636.

46. Kurapati KR, Atluri VS, Samikkannu T, Nair MP. Ashwagandha (Withania somnifera) reverses beta-amyloid1-42 induced toxicity in human neuronal cells: implications in HIV-associated neurocognitive disorders (HAND). PLoS One. 2013;8(10):e77624.

47. Agudelo M, Gandhi N, Saiyed Z, et al. Effects of alcohol on histone deacetylase 2 (HDAC2) and the neuroprotective role of trichostatin A (TSA). Alcohol Clin Exp Res. 2011;35(8):1550-1556.

48. Zhang Y, Wang M, Li H, et al. Accumulation of nuclear and mitochondrial DNA damage in the frontal cortex cells of patients with HIVassociated neurocognitive disorders. Brain Res. 2012;1458:1-11.

49. Louboutin JP, Strayer D. Role of oxidative stress in HIV-1-associated neurocognitive disorder and protection by gene delivery of antioxidant enzymes. Antioxidants (Basel). 2014;3(4):770-797.

50. Tejima E, Guo S, Murata Y, et al. Neuroprotective effects of overexpressing tissue inhibitor of metalloproteinase TIMP-1. J Neurotrauma. 2009;26(11):1935-1941.
51. Dhawan S, Weeks BS, Soderland C, et al. HIV-1 infection alters monocyte interactions with human microvascular endothelial cells. J Immunol. 1995;154(1):422-432.

52. Suryadevara R, Holter S, Borgmann K, et al. Regulation of tissue inhibitor of metalloproteinase-1 by astrocytes: links to HIV-1 dementia. Glia. 2003;44(1):47-56.

53. Jourquin J, Tremblay E, Bernard A, et al. Tissue inhibitor of metalloproteinases-1 (TIMP-1) modulates neuronal death, axonal plasticity, and learning and memory. Eur J Neurosci. 2005;22(10):2569-2578.

54. Yong VW, Power C, Forsyth P, Edwards DR. Metalloproteinases in biology and pathology of the nervous system. Nat Rev Neurosci. 2001;2(7):502-511.

55. Nedivi E, Hevroni D, Naot D, Israeli D, Citri Y. Numerous candidate plasticity-related genes revealed by differential cDNA cloning. Nature. 1993;363(6431):718-722.

56. Pilakka-Kanthikeel S, Atluri VSR, Sagar V, Saxena SK, Nair M. Targeted brain derived neurotropic factors (BDNF) delivery across the blood-brain barrier for neuro-protection using magnetic nano carriers: an in-vitro study. PLoS One. 2013;8(4):e62241.

57. Mastroianni CM, Liuzzi GM. Matrix metalloproteinase dysregulation in HIV infection: implications for therapeutic strategies. Trends $\mathrm{Mol}$ Med. 2007;13(11):449-459.

58. Hudson L, Liu J, Nath A, et al. Detection of the human immunodeficiency virus regulatory protein tat in CNS tissues. J Neurovirol. 2000;6(2): $145-155$.

59. Latronico T, Liuzzi GM, Riccio P, et al. Antiretroviral therapy inhibits matrix metalloproteinase-9 from blood mononuclear cells of HIVinfected patients. AIDS. 2007;21(6):677-684

60. Nel A, Xia T, Mädler L, Li N. Toxic potential of materials at the nanolevel. Science. 2006;311(5761):622-627.

61. Diana V, Bossolasco P, Moscatelli D, Silani V, Cova L. Dose dependent side effect of superparamagnetic iron oxide nanoparticle labeling on cell motility in two fetal stem cell populations. PLoS One. 2013;8(11):e78435.

62. Velazquez I, Plaud M, Wojna V, Skolasky R, Laspiur JP, Melendez LM. Antioxidant enzyme dysfunction in monocytes and CSF of Hispanic women with HIV-associated cognitive impairment. J Neuroimmunol. 2009;206(1-2):106-111.

63. Viviani B, Corsini E, Binaglia M, Galli CL, Marinovich M. Reactive oxygen species generated by glia are responsible for neuron death induced by human immunodeficiency virus-glycoprotein 120 in vitro. Neuroscience. 2001;107(1):51-58.
International Journal of Nanomedicine

\section{Publish your work in this journal}

The International Journal of Nanomedicine is an international, peerreviewed journal focusing on the application of nanotechnology in diagnostics, therapeutics, and drug delivery systems throughout the biomedical field. This journal is indexed on PubMed Central, MedLine, CAS, SciSearch $®$, Current Contents ${ }^{\circledR} /$ Clinical Medicine,

\section{Dovepress}

Journal Citation Reports/Science Edition, EMBase, Scopus and the Elsevier Bibliographic databases. The manuscript management system is completely online and includes a very quick and fair peer-review system, which is all easy to use. Visit http://www.dovepress.com/ testimonials.php to read real quotes from published authors. 